\title{
Dirac Relaxation of the Israel Junction Conditions: Unified Randall-Sundrum Brane Theory
}

\author{
Aharon Davidson $*$ and Ilya Gurwich \\ Physics Department, Ben-Gurion University, Beer-Sheva 84105, Israel
}

(Dated: June 18, 2006)

\begin{abstract}
Following Dirac's brane variation prescription, the brane must not be deformed during the variation process, or else the linearity of the variation may be lost. Alternatively, the variation of the brane is done, in a special Dirac frame, by varying the bulk coordinate system itself. Imposing appropriate Dirac style boundary conditions on the constrained 'sandwiched' gravitational action, we show how Israel junction conditions get relaxed, but remarkably, all solutions of the original Israel equations are still respected. The Israel junction conditions are traded, in the $Z_{2}$-symmetric case, for a generalized Regge-Teitelboim type equation (plus a local conservation law), and in the generic $Z_{2}$-asymmetric case, for a pair of coupled Regge-Teitelboim equations. The Randall-Sundrum model and its derivatives, such as the Dvali-Gabadadze-Porrati and the Collins-Holdom models, get generalized accordingly. Furthermore, Randall-Sundrum and Regge-Teitelboim brane theories appear now to be two different faces of the one and the same unified brane theory. Within the framework of unified brane cosmology, we examine the dark matter/energy interpretation of the effective energy/momentum deviations from General Relativity.
\end{abstract}

\section{INTRODUCTION}

In an almost forgotten paper 1] entitled "An extensible model of the electron", Dirac has made an attempt to picture a classical spinless electron as a breathing bubble in the electromagnetic field 'with no constraints fixing its size and shape'. Some positive surface tension has been invoked in order 'to prevent the electron from flying apart under the Coulomb repulsion of it surface charge'. On the practical side, this naive model has not made any impact on particle physics, so in this paper, we make no use of the model itself. On the field theoretical side, however, although gravity was switched off in this paper, it was nonetheless the first brane model. The equations of motion are derivable from an action principle, and in particular, the model offers a detailed prescription how to consistently perform brane variation. In the present paper, we switch on gravity, and apply the Dirac brane variation prescription to modern brane theories.

Our main result is that the Israel[2] junction conditions (IJC), which are known to play a central role in all modern brane theories, get in fact relaxed. While every IJC solution is still strictly respected, it represents now a whole (continuous) family of new solutions. This opens the door for brane unification. To be more specific, as schematically illustrated in Fig प both ReggeTeitelboim [3] and Randall-Sundrum [4] brane theories, which generalize General Relativity in very different theoretical directions, appear to be two faces of the one and the same unified brane theory. Also represented in the scheme are Dirac model along with Dvali-GabadadzePorrati [5] and Collins-Holdom [6] models. These latter brane models switch on the Randall-Sundrum repressed

\footnotetext{
*Email: davidson@bgu.ac.il

†Email: gurwichphys@gmail.com
}

$\mathcal{R}_{4}$ brane curvature term.

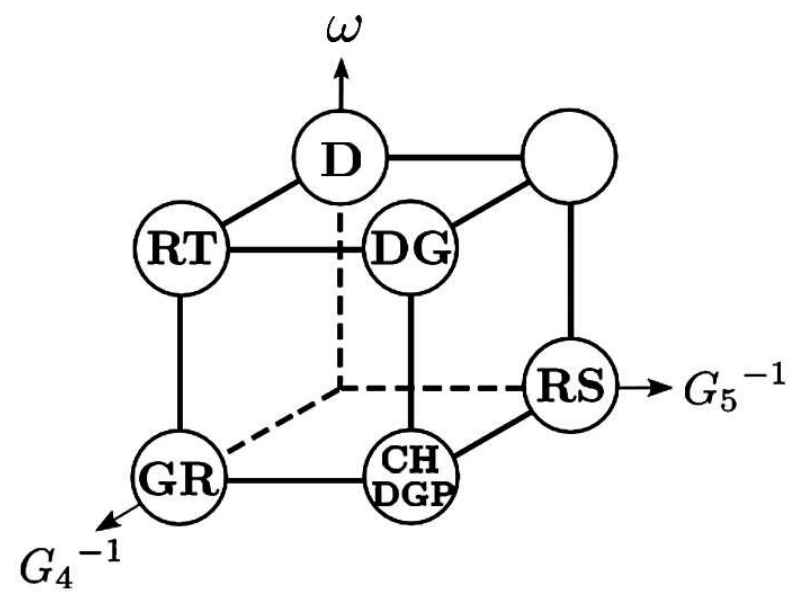

FIG. 1: Randall-Sundrum (RS) and Regge-Teitelboim (RT) brane theories, which generalize General Relativity (GR), appear to be two different faces of the one and the same unified brane theory (DG). $\omega$ denotes a Regge-Teitelboim constant of integration. Also represented in the scheme are Dirac's extensible model (D), and the Dvali-Gabadadze-Porrati (DGP) and Collins-Holdom $(\mathrm{CH})$ extensions of the RS model.

The Randall-Sundrum theory is very well known, and has rightly attracted lots of attention from General Relativity 7], cosmology [8] in particular, and also from string theory [9] points of view. See Ref. 10] for some brane world reviews, and Ref. 11] for some earlier brane models. The much older Regge-Teitelboim theory, on the other hand, a generalized Nambu-Goto type brane theory [] for quantum gravity, has remained quite unfamiliar. This is partially due to the fact that the theory was originally demonstrated within the naive framework of a flat (rather than AdS) non-dynamical (rather than dynamical) higher dimensional background. The Regge-Teitelboim theory suffered some criticism 12 in 
the past, but given its built-in Einstein limit and a handful of attractive features 13, 14] (in particular, a dark companion 13 to any energy density, a quadratic Hamiltonian formalism 15], and a rather novel approach to brane nucleation [16]), it offers a unique deviation from General Relativity. The associated so-called Geodesic Brane Gravity (GBG) is conveniently parametrized by a conserved 'bulk energy' (see $\omega$ in Fig.1) which fades away at the Einstein limit. Invoking the Dirac brane variation prescription, the appropriately generalized ReggeTeitelboim theory naturally becomes the $G_{5} \rightarrow \infty$ limit of the unified Randall-Sundrum brane theory.

To see how all this comes about, let us first 'listen' very carefully to Dirac. Consider thus a flat 4-dimensional Minkowski background

$$
d s^{2}=-d t^{2}+d r^{2}+r^{2} d \Omega^{2},
$$

and denote the breathing radius of the Dirac bubble by $r=f(t)$. Alternatively, perform an implicit general coordinate transformation $R=r-f(t)$, such that in the new frame, with metric

$$
d s^{2}=-d t^{2}+(d R+\dot{f} d t)^{2}+(R+f(t))^{2} d \Omega^{2},
$$

the bubble location, conveniently set at $R=0$, does not change during the variation process. In both frames of reference the unknown function $f(t)$ is to be determined by apparently minimizing the same Lagrangian, so what is the difference? According to Dirac, the trouble lies with the fact that the inner $(R<0)$ and the outer $(R>0)$ regions of the bubble are not a smooth continuation of each other. In particular, originating from a close surface electric charge distribution, the associated electric field solely lives outside the bubble. To be a bit more quantitative, introduce parameters $u_{1,2,3}$ to specify a general point on the bubble, and consider the embedding vector $x^{\mu}(u)$ as a canonical variable. The alarming point is that the variation $\delta I$ is not necessarily a linear function of $\delta x^{\mu}$. Quoting Dirac, 'If one makes a variation $\delta x^{\mu}$ corresponding to the surface being pushed out a little, $\delta I$ will not be minus the $\delta I$ for $-\delta x^{\mu}$, corresponding to the surface being pushed in a little, on account of the field just outside the surface being different from the field just inside. Thus this choice of canonical variables will not $\boldsymbol{d o}$ '. To bypass the problem, Dirac has ingeniously introduced general curvilinear coordinates, such that in the new coordinate system, the location of the bubble does not change during the variation process. The general idea being 'to produce an arbitrary variation of the surface by varying the coordinate system'. Modern brane theories based on an action principle better follow this prescription or else the linearity, and hence the self-consistency, of the variation may be in jeopardy.

Although our paper is about relaxing the Israel junction conditions, it is worth while mentioning the BattyeCarter approach [17, where the brane junction conditions are in fact further constrained. In their model, the Israel junction conditions are not only kept alive, but are furthermore supplemented by a force equation. The BattyeCarter force equation is trivially satisfied, however, when $Z_{2}$ symmetry is enforced by hand. In the present paper, for comparison, the Israel junction conditions get relaxed, and the net effect does not die away at the $Z_{2}$ limit.

The introduction was mainly devoted to getting acquainted with the Dirac brane variation. The rest of the paper is organized as follows. In Section 2, we covariantly formulate the Dirac brane conditions. In section 3, we support the Dirac brane prescription by an explicit action principle (invoking a set of Lagrange multipliers to manifestly deal with the various constraints floating around), and carefully perform the variation without deforming the brane, and without changing its location. Next, Section 4 is where the Israel Junction Conditions connect with the Regge-Teitelboim theory, and get accordingly relaxed. A detailed general analysis is offered without appealing to a specific (say) $Z_{2}$ symmetry. Unified brane cosmology is discussed in Section 5. One integration of the equations of motion can be carried out analytically, thereby introducing the novel constant of integration $\omega$, which parametrizes the deviation from the Randall-Sundrum model and of course from General Relativity. In Section 6, the various limits of the theory (including in particular General Relativity, RandallSundrum, and Regge-Teitelboim limits) are being discussed in some details. And finally, we summarize the paper by drawing some critical conclusions, and sketching some exciting paths for future research.

\section{GRAVITATIONAL EXTENSION OF DIRAC'S BOUNDARY CONDITIONS}

Had we considered a curved, yet non-dynamical, background (allow for different metrics $i=L, R$ on the two sides of the brane), the discussion would have been very much the same. Following Dirac, given some fixed bulk metrics $G_{A B}^{i}(y)$, the naive use of the embedding vectors $y^{i A}(x)$ as canonical fields is physically inconsistent. Alternatively, perform first the implicit general coordinate transformations $y^{i A} \rightarrow z^{i a}(y)$, such that in the new Dirac $z$-frame, with bulk metrics

$$
G_{a b}^{i}(z)=G_{A B}^{i}(z(y)) y_{, a}^{i A} y_{, b}^{i B},
$$

the brane is kept at rest during the variation process. The corresponding variations, namely

$$
\delta_{1} G_{a b}^{i}=G_{A B, C}^{i} \delta y^{i C} y_{, a}^{i A} y_{, b}^{i B}+2 G_{A B}^{i} y_{, a}^{i A} \delta y_{, b}^{i B},
$$

do not deform the brane.

When bulk gravity is finally switched on, however, the gravitational fields $G_{A B}^{i}$ themselves may vary (in addition to the above variation associated with $\delta y^{i A}$ ), so there are now two different contributions to $\delta G_{a b}$, namely

$$
\delta G_{a b}=\delta_{1} G_{a b}+\delta_{2} G_{a b},
$$


where

$$
\delta_{2} G_{a b}=y_{, a}^{A} y_{, b}^{B} \delta G_{A B} .
$$

It is well known that, on the bulk, the arbitrariness of $\delta G_{A B}$ leads to Einstein equations, whereas the arbitrariness of $\delta y^{A}$, the essence of re-parametrization covariance, is actually 'swallowed' in the sense that it does not lead to any field equation. But this by itself does not necessarily mean that the variation must stay fully arbitrary on the brane as well. In fact, we now argue that a fully arbitrary $\left.\delta G_{a b}\right|_{\text {brane }}$ would not only drive the Dirac frame meaningless, but would furthermore violate Dirac's 'linearity of the variation' principle.

As long as $\left.\delta G_{a b}\right|_{b r a n e}$ is general enough and is not restricted (to be more specific, it contains the full ten degrees of freedom), the corresponding equation of motion, symbolically written in the form

$$
\left.\frac{\delta \mathcal{L}}{\delta G_{a b}}\right|_{\text {brane }}=0
$$

would strictly hold irrespective of the $\left.\delta_{1} G_{a b}\right|_{\text {brane }}$ contribution. This would practically close the door for performing a consistent Dirac style $\delta y^{A}$ brane variation. An elegant way out, and may be the only covariant way out, is to impose the following (to be referred to as) Dirac boundary conditions

$$
\left.\delta_{2} G_{a b}\right|_{\text {brane }}=0
$$

such that, on the brane, one would only tolerate the arbitrariness of $\left.\delta_{1} G_{a b}\right|_{b r a n e}$. The latter condition, which keeps the brane not deformed during the variation process, is however not just a matter of choice or convenience. It is in fact mandatory. Rephrasing Dirac's argument, if Eq. (8) is violated, a tiny deformation of the brane corresponding to the brane being pushed a little to the right will not be minus the variation corresponding to the brane being pushed a little (equally) to the left, on account of the $L, R$ bulk sections not being a smooth continuation of each other. Eq. (8) paves thus the way for Dirac relaxation of Israel junction conditions. It should be noted that an earlier attempt 18] to carry out brane variation Dirac style has unfortunately failed to impose the boundary conditions Eq.(8), and consequently the Israel junction conditions, although derived, were not relaxed.

A important remark is now in order. In general, there are matter fields living in the bulk, and obviously, their variation (in Dirac's $z$-frame) consists of two parts. For example, associated with some (say) vector field

$$
V_{a}(z)=V_{A}(z(y)) y_{, a}^{A},
$$

is the variation

$$
\left.\delta V_{a}\right|_{b u l k}=\delta V_{A} y_{, a}^{A}+V_{A, B} \delta y^{B} y_{, a}^{A}+V_{A} \delta y_{, a}^{A} .
$$

Does it mean, in some (false) analogy with Eq.(8), that one is obliged to further impose

$$
\left.\delta_{2} V_{a}\right|_{\text {brane }}=\left.y_{, a}^{A} \delta V_{A}\right|_{\text {brane }}=0 ?
$$

Certainly not. The gravitational field $G_{a b}$ is the only field in the theory that, when being arbitrarily varied, it actually deforms the brane. Once the brane is consistently put to rest during the variation process, meaning Eq. (8) is fulfilled, Dirac's 'linearity of the variation' principle is fully respected. In turn, all matter fields may freely vary on the brane, and thus, all conventional non-gravitational matching conditions stay intact.

\section{DIRAC-STYLE BRANE VARIATION}

Let the bulk line elements, on the two sides $(i=L, R)$ of the brane, be respectively

$$
d s_{i}^{2}=G_{A B}^{i}(y) d y^{i A} d y^{i B} .
$$

The brane metric gets then fixed once the two sets of embedding coordinates $y^{i A}\left(x^{\mu}\right)$ are consistently specified, so that the common brane metric takes the form

$$
g_{\mu \nu}(x)=G_{A B}^{L}(y(x)) y_{, \mu}^{L A} y_{, \nu}^{L B}=G_{A B}^{R}(y(x)) y_{, \mu}^{R A} y_{, \nu}^{R B} .
$$

The fact that the brane metric is induced, rather than fundamental, is crucial. One may rightly conclude that, in a constrained brane gravity theory, unlike in General Relativity, $g_{\mu \nu}(x)$ cannot serve as a canonical field variable in the underlying Lagrangian formalism. Alternatively, the role of canonical fields is then naturally taken by the embedding vectors $y^{i A}\left(x^{\mu}\right)$, bearing in mind that their physically consistent variation must be carried out Dirac style. Needless to say, if the higher dimensional background is furthermore dynamical, the metric tensors $G_{A B}^{L, R}(y)$ enter the game as additional canonical fields.

The prototype brane action is the following

$$
\begin{aligned}
I & =\int_{L}\left(-\frac{1}{16 \pi G_{5}} \mathcal{R}^{L}+\mathcal{L}_{m}^{L}\right) \sqrt{-G_{L}} d^{5} y+ \\
& +\frac{1}{8 \pi G_{5}} \int \mathcal{K}^{L} \sqrt{-g} d^{4} x+ \\
& +\int\left(-\frac{1}{16 \pi G_{4}} \mathcal{R}+\mathcal{L}_{m}\right) \sqrt{-g} d^{4} x+ \\
& +\frac{1}{8 \pi G_{5}} \int \mathcal{K}^{R} \sqrt{-g} d^{4} x+ \\
& +\int_{R}\left(-\frac{1}{16 \pi G_{5}} \mathcal{R}^{R}+\mathcal{L}_{m}^{R}\right) \sqrt{-G_{R}} d^{5} y
\end{aligned}
$$

The constrained gravity brane Lagrangian is 'sandwiched' between the left and the right general relativistic bulk Lagrangians (an early version of such a 'sandwich' Lagrangian, describing a cosmic solenoid, was considered 
in Ref.[19]). The presence of the Gibbons-Hawking 21] boundary terms here is known to be mandatory, not optional. It is well known that without this exact term there is no way to integrate out all $\delta G_{A B ; C}$ terms on the brane. In our notations,

$$
\mathcal{K}=g^{\mu \nu} \mathcal{K}_{\mu \nu}=P^{A B} \mathcal{K}_{A B}
$$

denotes the scalar extrinsic curvature,

$$
\begin{gathered}
\mathcal{K}_{\mu \nu}=\frac{1}{2} y_{, \mu}^{A} y_{, \nu}^{B}\left(n_{A ; B}+n_{B ; A}\right)= \\
=\frac{1}{2}\left(y_{, \mu}^{A} n_{A, \nu}+y_{, \nu}^{A} n_{A, \mu}-2 \Gamma_{B C}^{A} y_{, \mu}^{B} y_{, \nu}^{C} n_{A}\right)
\end{gathered}
$$

is the extrinsic curvature tensor,

$$
P^{A B}=G^{A B}-n^{A} n^{B}=g^{\mu \nu} y_{, \mu}^{A} y_{, \nu}^{B}
$$

is the projection tensor (also known as the induced metric), and $n^{A}$ being the pointing outwards space-like unit normal to the brane. The induced brane metric itself is given by both

$$
g_{\mu \nu}=G_{A B}^{L} y_{, \mu}^{L A} y_{, \nu}^{L B}=G_{A B}^{R} y_{, \mu}^{R A} y_{, \nu}^{R B} .
$$

Notice that in the Lagrangian specified by Eq.(14), we keep (i) The Randall-Sundrum option of throwing away the 4-dimensional Ricci scalar $\mathcal{R}$ (by letting $G_{4} \rightarrow \infty$ ), and (ii) The Regge-Teitelboim option of turning the higher dimensional background non-dynamical (by letting on $\left.G_{5} \rightarrow \infty\right)$.

The canonical fields associated with the above action, are $G_{A B}^{L, R}(y)$, and the brane location $y^{A}(x)$ (to be treated as prescribed by Dirac). At the technical level, however, given the various constrains floating around, e.g. Eq.(18), we find this Lagrangian too complicated to handle. Thus, to make the constraints manifest, we invoke a set of Lagrange multipliers, and correspondingly add an extra piece $I_{c o n}$ to the action, namely

$$
\begin{aligned}
& I_{\text {con }}=\sum_{i=L, R}\left[\int \lambda_{i}^{\mu \nu}\left(g_{\mu \nu}-G_{A B}^{i} y_{, \mu}^{i A} y_{, \nu}^{i B}\right)+\right. \\
& \left.+\eta_{i}^{\mu} y_{, \mu}^{i A} n_{A}^{i}+\sigma_{i}\left(G_{A B}^{i} n^{i A} n^{i B}-1\right)\right] \sqrt{-g} d^{4} x
\end{aligned}
$$

In this alternative formalism, $\lambda_{L, R}^{\mu \nu}, \eta_{L, R}^{\mu}$, and $\sigma_{L, R}$ denote Lagrange multipliers, whereas $G_{A B}^{L, R}(y), n_{A}^{L, R}(y)$, and $g_{\mu \nu}(x)$ are considered independent canonical fields. In particular, it is crucial to emphasize that the embedding vectors $y_{L}^{A}(x)$ and $y_{R}^{A}(x)$ (like $n_{L}^{A}$ and $n_{R}^{A}$ ) are kept independent at the Lagrangian level, leading to two field equations. It is only when the constrains associated with the Lagrange multipliers $\lambda_{\mu \nu}^{L, R}$ are written down explicitly, at the equation of motion level, that the two metrics $g_{\mu \nu}^{L, R}(x)$, induced by $y_{L}^{A}(x)$ and $y_{R}^{A}(x)$ respectively, become in fact the one and the same brane metric $g_{\mu \nu}(x)$.

We now perform the variation in steps:
- The variation of $I+I_{\text {con }}$ with respect to the normals $n_{A}^{L, R}$ leads to the field equation (for $L, R$ separately)

$$
-\frac{1}{8 \pi G_{5}} g^{\mu \nu}\left(y_{; \mu \nu}^{A}+\Gamma_{B C}^{A} y_{, \mu}^{B} y_{, \nu}^{C}\right)+\eta^{\mu} y_{, \mu}^{A}+2 \sigma n^{A}=0 \text {. }
$$

To squeeze its contents, one may project this equation on the brane (i.e. multiplying by $\left.G_{D A} y_{, \lambda}^{D}\right)$ to learn that

$$
\eta_{\mu}=0
$$

and also perpendicular to the brane (i.e. multiplying by $\left.n_{A}\right)$ to find out that

$$
\sigma=-\frac{1}{16 \pi G_{5}} \mathcal{K} .
$$

- The variation of $I+I_{\text {con }}$ with respect to the brane metric $g_{\mu \nu}$ contains contributions from both $i=L, R$, and after taking the various constraints into account, one is left with the field equation

$$
\begin{gathered}
\frac{1}{16 \pi G_{4}}\left(\mathcal{R}^{\mu \nu}-\frac{1}{2} g^{\mu \nu} \mathcal{R}\right)+\frac{1}{2} \mathcal{T}^{\mu \nu}+ \\
+\sum_{i=L, R} \lambda_{i}^{\mu \nu}-\frac{1}{8 \pi G_{5}} \sum_{i=L, R}\left(\mathcal{K}_{i}^{\mu \nu}-\frac{1}{2} g^{\mu \nu} \mathcal{K}_{i}\right)=0 .
\end{gathered}
$$

- The bulk variation of $I+I_{\text {con }}$ with respect to the metrics $G_{a b}^{L, R}$ is next. It is clear from our notations that, at this stage, it is already mandatory to follow the Dirac prescription. A useful formula here is the contraction of a general tensor $E^{a b}$ with the variation of the metric $\delta G_{a b}$

$$
\begin{gathered}
E^{a b} \delta G_{a b}=E^{A B} \delta G_{A B}- \\
-2\left(E^{A B}\right)_{; B} G_{A D} \delta y^{D}+2\left(E^{a b} G_{A B} y_{, a}^{A} \delta y^{B}\right)_{; b} .
\end{gathered}
$$

In particular, applying the above to

$$
E_{i}^{a b}=\frac{1}{8 \pi G_{5}}\left(\mathcal{R}_{i}^{a b}-\frac{1}{2} G_{i}^{a b} \mathcal{R}_{i}\right)+\mathcal{T}_{i}^{a b},
$$

we learn that there are no surprises in the two $i=L, R$ bulk sections, which are conventionally governed by the 5-dimensional Einstein equations $E_{i}^{A B}=0$. Notice that the total derivative term in Eq. (24) can be transformed into a boundary term by means of Stokes's theorem, and thus may in principle contribute to the $\delta G_{a b}$ variation on the brane. But such a contribution clearly vanishes due to the fact that on the bulk Einstein equations are satisfied.

- The variation of $I+I_{\text {con }}$ with respect to the metrics $\left.G_{a b}^{L, R}\right|_{\text {brane }}$ (for $L, R$ separately) is where Dirac boundary conditions Eq. (8) are expected to make their impact. Given the above action principle, one can now explicitly specify the corresponding integrant, namely

$$
\begin{aligned}
\left.\frac{1}{\sqrt{-g}} \frac{\delta \mathcal{L}}{\delta G_{a b}}\right|_{\text {brane }} & =\frac{1}{16 \pi G_{5}}\left[n^{a} g^{\mu \nu}\left(y_{; \mu \nu}^{b}+\Gamma_{c d}^{b} y_{, \mu}^{c} y_{, \nu}^{d}\right)+\right. \\
& \left.+n_{; c}^{a} P^{b c}\right]-\lambda^{\mu \nu} y_{, \mu}^{a} y_{, \nu}^{b}-\sigma n^{a} n^{b} .
\end{aligned}
$$


Had we equated the RHS of Eq.(26) to zero, which is however not the case here, the normal-normal component $\frac{\delta \mathcal{L}}{\delta G_{a b}} n_{a} n_{b}$ would have re-produced Eq.(22), the normal-tangent components $\frac{\delta \mathcal{L}}{\delta G_{a b}} G_{a c} y_{, \alpha}^{c} n_{b}$ would have vanished identically, and the tangent-tangent components $\frac{\delta \mathcal{L}}{\delta G_{a b}} G_{a c} y_{, \alpha}^{c} G_{b d} y_{, \beta}^{d}$ would have resulted in

$$
\frac{1}{16 \pi G_{5}} \mathcal{K}_{\mu \nu}-\lambda_{\mu \nu}=0
$$

thereby taking us directly, see Eq.(23), to Israel junction conditions.

At this point, we find it quite amazing to recall that the Israel junction conditions [2] were originally introduced without the support of an underlying action principle. For some early derivation of IJC from a gravitational action principle, see for example Ref. 19], and Ref. [20] for a dilatonic version and other extensions.

However, as argued earlier, insisting on Dirac boundary conditions Eq.(8), the RHS of Eq.(26) does not necessarily vanish. To derive the modified field equation, we first substitute $\sigma$, as given by Eq.(22), so that the variation on the brane takes a more compact form

$$
\left.\int \sqrt{-g}\left(\frac{1}{16 \pi G_{5}} \mathcal{K}^{a b}-\lambda^{\mu \nu} y_{, \mu}^{a} y_{, \nu}^{b}\right) \delta_{1} G_{a b}\right|_{\text {brane }} d^{4} x=0 .
$$

We know that now, once $\sigma$ has been already substituted, the normal-normal component of the tensor in parentheses vanishes, while its normal-tangent components vanish identically. Thus, the only contribution to the integral comes from the tangent-tangent components, namely

$$
\left.\int \sqrt{-g}\left(\frac{1}{16 \pi G_{5}} \mathcal{K}^{\mu \nu}-\lambda^{\mu \nu}\right) \delta_{1} G_{a b}\right|_{\text {brane }} y_{, \mu}^{a} y_{, \nu}^{b} d^{4} x=0 \text {. }
$$

Expressing $\left.\delta_{1} G_{a b}\right|_{\text {brane }}$ in terms of $\delta y^{A}$ and its derivatives, see Eq.(4), we are finally led to

$$
\begin{aligned}
& \int \sqrt{-g}\left(\frac{1}{16 \pi G_{5}} \mathcal{K}^{\mu \nu}-\lambda^{\mu \nu}\right) \\
& \left(G_{A B, C} y_{, \mu}^{A} y_{, \nu}^{B} \delta y^{C}+2 G_{A B} y_{, \mu}^{A} \delta y_{, \nu}^{B}\right) d^{4} x=0 .
\end{aligned}
$$

At this stage, no trace is left from the Dirac frame (that is to show that it is no more than a mathematical tool), and the variation acquires the familiar Regge-Teitelboim form. It is now straight forwards to integrate by parts, and verify that the arbitrariness of $\delta y^{A}$ gives rise to

$$
\begin{gathered}
{\left[\left(\frac{1}{16 \pi G_{5}} \mathcal{K}^{\mu \nu}-\lambda^{\mu \nu}\right) y_{, \mu}^{A}\right]_{; \nu}+} \\
+\Gamma_{B C}^{A}\left(\frac{1}{16 \pi G_{5}} \mathcal{K}^{\mu \nu}-\lambda^{\mu \nu}\right) y_{, \mu}^{B} y_{, \nu}^{C}=0,
\end{gathered}
$$

for $i=L, R$ separately. Reflecting a fundamental embedding identity, the velocity $y_{, \mu}^{A}$ and the covariant acceleration $y_{; \mu \nu}^{A}+\Gamma_{B C}^{A} y_{, \mu}^{B} y_{, \nu}^{C}$, viewed as vectors on the bulk, are orthogonal to each other. In turn, Eq. (31) splits into two parts, each of which must vanish separately. Whereas the part proportional to $y_{, \mu}^{A}$ implies the local conservation law

$$
\left(\frac{1}{16 \pi G_{5}} \mathcal{K}^{\mu \nu}-\lambda^{\mu \nu}\right)_{; \nu}=0
$$

the second part is the geodesic brane equation

$$
\left(\frac{1}{16 \pi G_{5}} \mathcal{K}^{\mu \nu}-\lambda^{\mu \nu}\right)\left(y_{; \mu \nu}^{A}+\Gamma_{B C}^{A} y_{, \mu}^{B} y_{, \nu}^{C}\right)=0 .
$$

Appreciating the fact that the extrinsic curvature is the normal component of the covariant acceleration, the latter equation takes the more geometric oriented form

$$
\left(\frac{1}{16 \pi G_{5}} \mathcal{K}_{\mu \nu}-\lambda_{\mu \nu}\right) \mathcal{K}^{\mu \nu}=0
$$

Altogether, the tensorial Eq.(27), associated with Israel junction conditions, is replaced by the $\mathcal{K}$-contracted scalar Eq.(34) plus the conservation law Eq.(32).

\section{RELAXING THE ISRAEL JUNCTION CONDITIONS}

Define the combined Einstein-Israel tensor

$$
\begin{gathered}
\mathcal{S}_{\mu \nu} \equiv \frac{1}{16 \pi G_{4}}\left(\mathcal{R}_{\mu \nu}-\frac{1}{2} g_{\mu \nu} \mathcal{R}\right)+ \\
+\frac{1}{2} \mathcal{T}_{\mu \nu}-\frac{1}{16 \pi G_{5}} \sum_{i=L, R}\left(\mathcal{K}_{\mu \nu}^{i}-\mathcal{K}^{i} g_{\mu \nu}\right) .
\end{gathered}
$$

In this convenient notation, our gravitational field equations take the compact form

$$
\mathcal{S}_{\mu \nu}=\sum_{i=L, R}\left(\frac{1}{16 \pi G_{5}} \mathcal{K}_{\mu \nu}^{i}-\lambda_{\mu \nu}^{i}\right)
$$

Now, if Dirac's brane variation procedure is ignored, then the RHS vanishes, and one recovers the well known Israel junction conditions $\mathcal{S}_{\mu \nu}=0$. However, if the Dirac brane variation procedure is adopted, the RHS does not necessarily vanish, and one is alternatively led to

$$
\begin{aligned}
& \left(\frac{1}{16 \pi G_{5}} \mathcal{K}_{\mu \nu}^{L}-\lambda_{\mu \nu}^{L}\right) \mathcal{K}_{L}^{\mu \nu}=0 \\
& \left(\frac{1}{16 \pi G_{5}} \mathcal{K}_{\mu \nu}^{R}-\lambda_{\mu \nu}^{R}\right) \mathcal{K}_{R}^{\mu \nu}=0
\end{aligned}
$$

accompanied by two independent conservation laws

$$
\begin{aligned}
& \left(\frac{1}{16 \pi G_{5}} \mathcal{K}_{L}^{\mu \nu}-\lambda_{L}^{\mu \nu}\right)_{; \nu}=0, \\
& \left(\frac{1}{16 \pi G_{5}} \mathcal{K}_{R}^{\mu \nu}-\lambda_{R}^{\mu \nu}\right)_{; \nu}=0 .
\end{aligned}
$$


Notice that Eqs.(3738) cause the master Eq.(36) to split into two projections, namely

$$
\begin{aligned}
& \mathcal{S}_{\mu \nu} \mathcal{K}_{L}^{\mu \nu}=\left(\frac{1}{16 \pi G_{5}} \mathcal{K}_{\mu \nu}^{R}-\lambda_{\mu \nu}^{R}\right) \mathcal{K}_{L}^{\mu \nu} \\
& \mathcal{S}_{\mu \nu} \mathcal{K}_{R}^{\mu \nu}=\left(\frac{1}{16 \pi G_{5}} \mathcal{K}_{\mu \nu}^{L}-\lambda_{\mu \nu}^{L}\right) \mathcal{K}_{R}^{\mu \nu}
\end{aligned}
$$

whereas the major role of Eqs.(3940), combined with the Coddazi relations, see Eq. [55], is to assure generalized energy/momentum conservation even though Einstein and Israel equations get relaxed.

Given the set of Eqs. (37.41), it is in general impossible to construct a generic brane equation free of the Lagrange multipliers $\lambda_{\mu \nu}^{L, R}$. However, there are two special cases for which the calculation of $\lambda_{\mu \nu}^{L, R}$ can be practically bypassed. These special cases are the following:

The smooth background case:

This 'mostly pedagogical' case is characterized by

$$
\mathcal{K}_{\mu \nu}^{L}+\mathcal{K}_{\mu \nu}^{R}=0
$$

so that

$$
\left(\lambda_{\mu \nu}^{L}+\lambda_{\mu \nu}^{R}\right) \mathcal{K}^{\mu \nu}=0 .
$$

Associated with this case is the original (to be referred to as the reduced) Regge-Teitelboim equation written in a geometrically oriented form [17]

$$
\left(\mathcal{R}_{\mu \nu}-\frac{1}{2} g_{\mu \nu} \mathcal{R}+8 \pi G_{4} \mathcal{T}_{\mu \nu}\right) \mathcal{K}^{\mu \nu}=0 .
$$

The Einstein limit, a crucial built-in feature of the ReggeTeitelboim theory, is manifest (for a finite $G_{4}$ ), with the Newton constant being easily identified as

$$
G_{N}=G_{4} .
$$

In particular, it is evident that every solution of Einstein equations is automatically a solution of the corresponding (reduced) Regge-Teitelboim equation. The deviation from General Relativity is expected to be parametrized by a novel constant of integration (details soon) .

The $Z_{2}$-symmetric case:

This 'more practical' case is characterized by

$$
\mathcal{K}_{\mu \nu}^{L}-\mathcal{K}_{\mu \nu}^{R}=0
$$

for which

$$
\left(\lambda_{\mu \nu}^{L}-\lambda_{\mu \nu}^{R}\right) \mathcal{K}^{\mu \nu}=0 .
$$

Associated with this case is the generalized (to be referred to as the full) Regge-Teitelboim equation

$$
\mathcal{S}_{\mu \nu} \mathcal{K}^{\mu \nu}=0
$$

where the Einstein-Israel tensor $\mathcal{S}_{\mu \nu}$ has been defined by Eq. (35). As expected, it manifestly exhibits a RandallSundrum limit, with or without the Einstein tensor contribution (which anyhow dies away as $G_{4} \rightarrow \infty$ ). In this limit, Following Collins-Holdom [ $\underline{6}$ ], one may rightly expect (to be re-derived later) the Newton constant to obey

$$
\frac{1}{G_{N}}=\frac{1}{G_{R S}}+\frac{1}{G_{4}},
$$

modifying its original Randall-Sundrum value

$$
G_{R S}=G_{5} \sqrt{-\frac{\Lambda_{5}}{6}} .
$$

Clearly, every solution of Israel junction conditions is now automatically a solution of the corresponding (full) Regge-Teitelboim equation. Also notice that we meet again the reduced Regge-Teitelboim equation at the limit where $G_{5} \rightarrow \infty$.

On pedagogical grounds, we find it convenient to define a asterisked energy/momentum tensor

$$
\mathcal{T}_{\mu \nu}^{*} \equiv \mathcal{T}_{\mu \nu}-\frac{1}{8 \pi G_{5}} \sum_{i=L, R}\left(\mathcal{K}_{\mu \nu}^{i}-\mathcal{K}^{i} g_{\mu \nu}\right)
$$

and asterisked Lagrange multipliers

$$
\lambda_{\mu \nu}^{i *} \equiv \lambda_{\mu \nu}^{i}-\frac{1}{8 \pi G_{5}} \mathcal{K}_{\mu \nu}^{i} .
$$

In this language, $\mathcal{T}_{\mu \nu}^{*}=0$ is recognized as the main brane equation in the original Randall-Sundrum model, where intrinsic brane gravity has been switched off $\left(G_{4} \rightarrow \infty\right) . \mathcal{T}_{\mu \nu}^{*}$ resumes a more natural role as an implicit energy/momentum tensor in the dressed (finite $G_{4}$ ) Randall-Sundrum model. Invoking the above definition, the full Regge-Teitelboim equation resembles its reduced (albeit asterisked) version which, for the $Z_{2}$ symmetric case of interest, takes the form

$$
\left(\mathcal{R}_{\mu \nu}-\frac{1}{2} g_{\mu \nu} \mathcal{R}+8 \pi G_{4} \mathcal{T}_{\mu \nu}^{*}\right) \mathcal{K}^{\mu \nu}=0 .
$$

Stemming from $\lambda_{\mu \nu}^{* i}$ conservation, the integrability condition of the resulting Regge-Teitelboim equation requires $\mathcal{T}_{\mu \nu}^{*}$ (not necessarily $\mathcal{T}_{\mu \nu}$ ) to be locally conserved on the brane

$$
\mathcal{T}_{; \nu}^{* \mu \nu}=0
$$

This is guaranteed in fact by Eqs. (3940), or more precisely by the Coddazi relation

$$
\begin{aligned}
\mathcal{T}_{; \nu}^{\mu \nu} & =\frac{1}{8 \pi G_{5}} \sum_{i=L, R}\left(\mathcal{K}_{i}^{\mu \nu}-\mathcal{K}_{i} g^{\mu \nu}\right)_{; \nu}= \\
& =\frac{1}{8 \pi G_{5}} g^{\mu \nu} \sum_{i=L, R} n_{i}^{a} \mathcal{R}_{a b}^{i} y_{, \nu}^{i b} .
\end{aligned}
$$

Given the special case of a maximally symmetric embedding spacetime (such as AdS), $\mathcal{T}_{\mu \nu}$ itself happens to be conserved. 


\section{UNIFIED BRANE COSMOLOGY}

In this section we deal with brane cosmology which is governed by a generic perfect fluid energy/momentum tensor. As usual, the latter is characterized by some energy density $\rho$, and isotropic pressure $P$. No specific equation of state $P=P(\rho)$ is chosen at this stage. The cosmological brane metric, which takes the standard FRW form

$$
d s^{2}=-d t^{2}+\frac{a^{2}(t)}{\left(1+\frac{1}{4} k r^{2}\right)^{2}} \delta_{i j} d x^{i} d x^{j},
$$

is embedded within a $Z_{2}$ symmetric AdS background with a negative cosmological constant $\Lambda_{5}<0$. The associated extrinsic curvatures are given explicitly by

$$
\mathcal{K}_{\mu \nu}^{L, R}=\left(\begin{array}{cc}
\frac{1}{\xi}\left(\frac{\ddot{a}}{a}-\frac{\Lambda_{5}}{6}\right) & 0 \\
0 & -\frac{a^{2} \xi}{\left(1+\frac{1}{4} k r^{2}\right)^{2}} \delta_{i j}
\end{array}\right)
$$

where we have used the convenient notation

$$
\xi \equiv \sqrt{\frac{\dot{a}^{2}+k}{a^{2}}-\frac{\Lambda_{5}}{6}} .
$$

Given the pair $(\rho, P)$ and the above expressions for $\mathcal{K}_{\mu \nu}^{L, R}$, we thus follow Eq.(51), and define the asterisked pair $\left(\rho^{*}, P^{*}\right)$ via

$$
\begin{aligned}
\rho^{*} & =\rho-\frac{3 \xi}{4 \pi G_{5}}, \\
P^{*} & =P+\frac{1}{4 \pi G_{5}}\left(2 \xi+\frac{1}{\xi}\left(\frac{\ddot{a}}{a}-\frac{\Lambda_{5}}{6}\right)\right) .
\end{aligned}
$$

We have already proven, see Eq.(54), the validity of the local conservation law

$$
\dot{\rho}^{*}+3 \frac{\dot{a}}{a}\left(\rho^{*}+P^{*}\right)=0
$$

which, owing to the maximally symmetric geometry of both bulk sections, is equivalent to

$$
\dot{\rho}+3 \frac{\dot{a}}{a}(\rho+P)=0
$$

The main equation for the FRW scale factor $a(t)$, namely Eq.(53), can now be put together, and one finds

$$
\begin{aligned}
& \left(8 \pi G_{4} \rho^{*}-3 \frac{\dot{a}^{2}+k}{a^{2}}\right) \mathcal{K}_{t t}+ \\
& +\frac{\left(1+\frac{1}{4} k r^{2}\right)^{2}}{a^{2}}\left(8 \pi G_{4} P^{*}+2 \frac{\ddot{a}}{a}+\frac{\dot{a}^{2}+k}{a^{2}}\right) \delta^{i j} \mathcal{K}_{i j}=0 .
\end{aligned}
$$

After some algebra, and $\mathcal{K}_{\mu \nu}$ substitution, it can be rearranged into

$$
\begin{aligned}
& \left(8 \pi G_{4} \rho^{*} a^{2}-3\left(\dot{a}^{2}+k\right)\right)\left(\ddot{a} a-\frac{\Lambda_{5}}{6} a^{2}\right)- \\
& -3\left(8 \pi G_{4} P^{*} a^{2}+2 \ddot{a} a+\left(\dot{a}^{2}+k\right)\right)\left(\dot{a}^{2}+k-\frac{\Lambda_{5}}{6} a^{2}\right)=0 .
\end{aligned}
$$

We have thus encountered a second order differential equation which we would now like to integrate out. To be more precise, recalling $\mathcal{T}_{\mu \nu}^{*}$ conservation and the fact that $\rho^{*}=\rho^{*}(\dot{a}, a)$, we are after some function

$$
F\left(\rho^{*}, \dot{a}, a\right)=\text { const. }
$$

The analytic answer [13] can be borrowed from the ReggeTeitelboim theory (only with $\rho^{*}$ replacing now $\rho$ ), namely

$$
F=a^{4}\left(3 \frac{\dot{a}^{2}+k}{a^{2}}-8 \pi G_{4} \rho^{*}\right) \sqrt{\frac{\dot{a}^{2}+k}{a^{2}}-\frac{\Lambda_{5}}{6}},
$$

and the same holds for the rest of the analysis. Consequently, it is very useful to define $\rho_{d}^{*}$ (to be referred to as the 'dark' companion of $\rho^{*}$ ) by means of

$$
\dot{a}^{2}+k \equiv \frac{8 \pi G_{4}}{3}\left(\rho^{*}+\rho_{d}^{*}\right) a^{2} .
$$

The justification for using here the word 'dark' is to be discussed soon. Plugging now the latter definition into $F\left(\rho^{*}, \dot{a}, a\right)$, one is immediately led to a the result

$$
\rho_{d}^{*^{2}}\left(8 \pi G_{4}\left(\rho^{*}+\rho_{d}^{*}\right)-\frac{\Lambda_{5}}{2}\right)=\frac{\omega^{2}}{a^{8}}
$$

where the novel constant of integration $\omega$ serves to parametrize the deviations from Randall-Sundrum brane cosmology.

Regarding the physical interpretation, imagine a physicist equipped with Einstein gravitational field equations, but totally ignorant of the existence of a fifth dimension and the associated brane gravity. Our physicist may have already calculated the Newton constant $G_{N}$, and is presumably capable of measuring the observed energy density $\rho(a)$. Verifying Einstein equations experimentally, that is establishing the ultimate interplay between geometry and matter/energy, is all what our physicist hopes for. Failing to do so consistently, however, with a discrepancy level of $90 \%$, he would presumably refer to the missing ingredient of General Relativity as $\rho_{\text {dark }}$, the so-defined illusive dark companion of observable $\rho$, thereby enforcing an effective FRW evolution based on

$$
\dot{a}^{2}+k \equiv \frac{8 \pi G_{N}}{3}\left(\rho+\rho_{\text {dark }}\right) a^{2} .
$$

It remains to be seen if unified brane gravity has anything to do with reality, but until then, one cannot resist the speculation that the missing dark matter is nothing but 
a brane artifact (various alternatives to dark matter and dark energy are reviewed in Ref. 22]). In other words, subject to the correct identification of $G_{N}$, unified brane gravity suggests

$$
\rho_{\text {dark }}=\frac{G_{4}}{G_{N}}\left(\rho^{*}+\rho_{d}^{*}\right)-\rho .
$$

Note that the idea of dark matter/energy unification [23], that is the possibility that artifact dark matter is nothing but the dark companion of $\rho=\Lambda_{4}$, been discussed within the framework of the original Regge-Teitelboim model.

Finally, we derive the cubic equation

$$
\frac{3 \xi^{3}}{8 \pi G_{4}}+\frac{3 \xi^{2}}{4 \pi G_{5}}+\left(\frac{\Lambda_{5}}{16 \pi G_{4}}-\rho(a)\right) \xi+\frac{\omega}{\sqrt{3} a^{4}}=0
$$

which directly relates $\xi$, as defined by Eq.(158), to the bare energy density $\rho(a)$. In turn, the FRW equation takes the effective form

$$
\frac{\dot{a}^{2}+k}{a^{2}}=\frac{\Lambda_{5}}{6}+\xi^{2}(a) .
$$

On the practical side, Eq. (70) allows us to conveniently navigate within the $\left(G_{4}^{-1}, G_{5}^{-1}, \omega\right)$ parameter space, with all special cases easily accessible.

\section{GR, RT, RS, DGP, AND CH LIMITS}

\section{A. Maximally symmetric brane}

To get a glimpse of what is lying ahead, let us first study the pedagogical case where the FRW cosmological brane evolution is enforced to be solely governed by a cosmological constant, namely

$$
\dot{a}^{2}+k=\frac{1}{3} \Lambda_{4} a^{2} .
$$

In which case, Eq. (66) immediately tells us that

$$
8 \pi G_{4}\left(\rho^{*}+\rho_{d}^{*}\right)=\Lambda_{4},
$$

which can be further translated, as dictated by Eq. (67), into

$$
\begin{aligned}
& \rho^{*}=\frac{\Lambda_{4}}{8 \pi G_{4}}+\frac{\omega}{a^{4} \sqrt{\Lambda_{4}-\frac{1}{2} \Lambda_{5}}}, \\
& \rho_{d}^{*}=-\frac{\omega}{a^{4} \sqrt{\Lambda_{4}-\frac{1}{2} \Lambda_{5}}}
\end{aligned}
$$

This simply means, following Eq.(59), that one should have actually started from the primitive energy density

$$
\rho(a)=\frac{\Lambda_{4}}{8 \pi G_{4}}+\frac{\sqrt{3\left(\Lambda_{4}-\frac{1}{2} \Lambda_{5}\right)}}{4 \pi G_{5}}+\frac{\omega}{a^{4} \sqrt{\Lambda_{4}-\frac{1}{2} \Lambda_{5}}},
$$

where the familiar Randall-Sundrum positive surface tension term is accompanied now by a less familiar, but quite characteristic, Regge-Teitelboim radiation-like term. It has been already demonstrated 24], within the framework of Regge-Teitelboim theory, that such a radiationlike term can in fact be of a dynamical origin. This calls, however, for a minimally coupled scalar field serendipitously accompanied by a quartic potential.

Several remarks are in order:

- For the records, the Regge-Teitelboim dark radiation term [13] was actually introduced before the RandallSundrum dark radiation term [25]. In spite of the similarity, however, the Regge-Teitelboim dark radiation does not seem to have anything to do with the RandallSundrum dark radiation. The latter enters the theory once the FRW brane is embedded [25] within (say) a Schwarzschild-AdS ${ }_{5}$ black hole background geometry.

- Keep in mind that $\omega$ is not necessarily a small quantity. Given the fact that $|\omega|$ parametrizes the deviation not only from Randall-Sundrum model but from General Relativity as well, its size will hopefully be fixed once the dark matter interpretation discussed earlier is fully established.

- At this stage, the sign of $\omega$ can still be either positive or negative. In the case of a maximally symmetric brane, for example, one observes that $\omega \rightarrow-\omega$ gives rise to $\rho_{d}^{*} \rightarrow-\rho_{d}^{*}$. More general, however, as far as the FRW cosmic evolution is concerned, one cannot really tell a theory associated with the pair $\left\{\rho^{*}, \rho_{d}^{*}\right\}$ from its dual based on $\left\{\rho^{*}+2 \rho_{d}^{*},-\rho_{d}^{*}\right\}$.

The flat Minkowski brane is of special interest. It is associated of course with setting $k=0$, and switching off $\Lambda_{4}$, but in unified brane gravity, it also requires starting from

$$
\rho(a)=\rho_{0}+\sqrt{\frac{2}{-\Lambda_{5}}} \frac{\omega}{a^{4}},
$$

where $\rho_{0}$ stands for the fine-tuned (to make $\Lambda_{4}$ vanish) Randall-Sundrum surface tension

$$
\rho_{0}=\frac{\sqrt{-6 \Lambda_{5}}}{8 \pi G_{5}} .
$$

Note that, whereas Randall-Sundrum fine-tuning of the brane tension is done at the Lagrangian level, the finetuning of the additional radiation-like term depends on the value of the would be constant of integration $\omega$.

To lift the radiation fine-tuning, consider next small energy density perturbations around a flat background, namely

$$
\rho=\left(\rho_{0}+\sqrt{\frac{2}{-\Lambda_{5}}} \frac{\omega}{a^{4}}\right)+\widetilde{\rho} .
$$


This clearly includes the special case $\widetilde{\rho}=\sqrt{\frac{2}{-\Lambda_{5}}} \frac{\Delta \omega}{a^{4}}$, but our interest in small $\widetilde{\rho}$ goes beyond this case. Let us thus define the small quantity $\epsilon$

$$
\epsilon=3 \frac{\dot{a}^{2}+k}{a^{2}}=8 \pi G_{4}\left(\rho^{*}+\rho_{d}^{*}\right)
$$

so that, following Eq. (67),

$$
\begin{aligned}
& \rho_{d}^{*} \simeq-\sqrt{\frac{2}{-\Lambda_{5}}} \frac{\omega}{a^{4}}\left(1+\frac{\epsilon}{\Lambda_{5}}\right) \\
& \rho^{*} \simeq \sqrt{\frac{2}{-\Lambda_{5}}} \frac{\omega}{a^{4}}\left(1+\frac{\epsilon}{\Lambda_{5}}\right)+\frac{\epsilon}{8 \pi G_{4}} .
\end{aligned}
$$

This is then substituted back into Eq.(159), and confronted with Eq.(79), and after some algebra one arrives at

$$
\widetilde{\rho}=\left(\frac{1}{8 \pi G_{4}}+\sqrt{\frac{6}{-\Lambda_{5}}}\left(\frac{1}{8 \pi G_{5}}+\frac{\omega}{\sqrt{3} \Lambda_{5} a^{4}}\right)\right) \epsilon .
$$

The fact that the coefficient of $\epsilon$ is not a constant means that we are away from General Relativity. It is only for large enough scale factors, namely for

$$
a(t) \gg\left(\frac{G_{5} \omega}{\Lambda_{5}}\right)^{1 / 4}
$$

that one faces the low-energy Randall-Sundrum limit, and the consequent identification Eq. (49) of the Newton constant. It has not escaped our attention that for tiny $a(t)$, much smaller than the above scale, a surplus amount $\Delta \omega$ of radiation would naturally lead to an effective brane cosmological constant $\Lambda_{4}^{\text {eff }}$ of order

$$
\Lambda_{4}^{e f f}=\mathcal{O}\left(\frac{\Delta \omega}{\omega} \Lambda_{5}\right)
$$

The question whether such a 'inflation from radiation' scenario can be fully matured, within the framework of a unified brane model, into a satisfactory theory of inflation is still open.

\section{B. Randall-Sundrum limit}

The Randall-Sundrum limit is automatically built-in in our theory. To study the Regge-Teitelboim deviation from the original Randall-Sundrum model, let us first solve Eq. (170) for $G_{4} \rightarrow \infty$. In which case, we find

$$
\xi=\frac{2 \pi G_{5}}{3}\left(\rho \pm \sqrt{\rho^{2}-\frac{\sqrt{3} \omega}{\pi G_{5} a^{4}}}\right) .
$$

The sign ambiguity of the quadratic equation can be removed by insisting on reproducing the Randall-Sundrum behavior at the $\omega \rightarrow 0$ limit. Choosing the tenable plus sign, we find

$$
\frac{\dot{a}^{2}+k}{a^{2}}=\frac{\Lambda_{5}}{6}+\left(\frac{2 \pi G_{5}}{3}\right)^{2}\left(\rho+\sqrt{\rho^{2}-\frac{\sqrt{3} \omega}{\pi G_{5} a^{4}}}\right)^{2}
$$

For small- $\omega, \xi$ can be approximated by

$$
\xi \simeq \frac{4 \pi G_{5}}{3} \rho-\frac{\omega}{\sqrt{3} \rho a^{4}},
$$

so that the Randall-Sundrum expansion starts with

$$
\frac{\dot{a}^{2}+k}{a^{2}} \simeq \frac{\Lambda_{5}}{6}+\left(\frac{4 \pi G_{5}}{3}\right)^{2} \rho^{2}-\frac{8 \pi G_{5} \omega}{3 \sqrt{3} a^{4}}
$$

In this limit, the effective energy density exhibits a so-called dark radiation term which highly reminds us of Randall-Sundrum cosmology in a radially symmetric Schwarzschild-AdS background. The corresponding 5dimensional Schwarzschild mass being

$$
m=-\frac{8 \pi G_{5}}{3 \sqrt{3}} \omega .
$$

However, one should keep in mind that the two sources of dark radiation are in fact independent of each other, and once our analysis is extended beyond $Z_{2}$ symmetry, both effects are expected to enter the game.

Once $G_{4}$ turns finite, and this is the generic case, Eq.(170) becomes cubic. Some algebra is then needed in order to single out the particular solution which is the analytic continuation of Eq. (877). Using the very convenient notation

$$
\chi(\rho) \equiv \sqrt{1-\frac{8 \pi G_{5}^{2}}{3 G_{4}}\left(\frac{\Lambda_{5}}{16 \pi G_{4}}-\rho\right)},
$$

assuming that $\rho$ is above some critical value (as otherwise another solution takes over), the singled out $\xi$ is approximated by

$$
\xi \simeq \frac{G_{4}}{G_{5}}(\chi-1)-\frac{4 \pi G_{5}^{2} \omega}{3 \sqrt{3} G_{4} \chi(\chi-1) a^{4}},
$$

so that the small- $\omega$ Collins-Holdom expansion starts now with

$$
\frac{\dot{a}^{2}+k}{a^{2}} \simeq \frac{\Lambda_{5}}{6}+\frac{G_{4}^{2}}{G_{5}^{2}}(\chi-1)^{2}-\frac{8 \pi G_{5} \omega}{3 \sqrt{3} \chi a^{4}} .
$$

In particular, for large enough $\rho(a)$, with the focus on the early Universe, not only is Einstein gravity met again (owing to $\chi \sim \sqrt{\rho}$ ), but the dark $\omega$-companion can be in fact very different from a simple dark radiation term. 


\section{Regge-Teitelboim limit}

Whereas the original Regge-Teitelboim limit is associated with letting $G_{5} \rightarrow \infty$ while keeping $\Lambda_{5}=0$, the modified Regge-Teitelboim limit still calls for $G_{5} \rightarrow \infty$, but the brane correctly separates now two tenable $\Lambda_{5}<0$ regions. Eq. (67), which is reduced now to

$$
\rho_{d}^{2}\left(8 \pi G_{4}\left(\rho+\rho_{d}\right)-\frac{\Lambda_{5}}{2}\right)=\frac{\omega^{2}}{a^{8}},
$$

can be used to directly assign a dark companion $\rho_{d}(\rho)$ to any given bare energy density $\rho$.

An apparently empty brane, characterized by $\rho=0$ leading to $\rho_{d} \neq 0$, is clearly a special yet a very pedagogical case. In which case, the non-vanishing dark energy density component behaves like

$$
\rho_{d}(a) \simeq \begin{cases}\left(\frac{\omega^{2}}{8 \pi G_{4} a^{8}}\right)^{1 / 3} & \rho_{d} \gg \frac{-\Lambda_{5}}{16 \pi G_{4}}, \\ \frac{\sqrt{2} \omega}{\sqrt{-\Lambda_{5}} a^{4}} & \rho_{d} \ll \frac{-\Lambda_{5}}{16 \pi G_{4}} .\end{cases}
$$

The natural scale emerging here is

$$
a_{c}^{4}=\frac{16 \sqrt{2} \pi G_{4} \omega}{\left(-\Lambda_{5}\right)^{3 / 2}} .
$$

At very early times $\left(a \ll a_{c}\right)$, the evolution is effectively governed by a mysterious $P_{\text {eff }}=-\frac{1}{9} \rho_{\text {eff }}$ negative pressure dark cosmic background. At late times $\left(a \gg a_{c}\right)$, on the other hand, it is the dark radiation which takes over. The inclusion of a brane cosmological constant in this game is straight forwards, and is easily achieved by shifting $\Lambda_{5} \rightarrow \Lambda_{5}-2 \Lambda_{4}$. One can then argue that the transition from a $P_{\text {eff }}=-\frac{1}{9} \rho_{\text {eff }}$ dominated Universe into a $\Lambda_{4}$ dominated Universe (accompanied by a leftover dark radiation of course) averagely resembles, at least at its early stages, an effective dark matter era, meaning $\rho_{d} \sim a^{-n}$ with $n \simeq 3$. Such a unified dark matter/energy 23] idea has already been demonstrated for the original $\left(\Lambda_{5}=0, G_{5} \rightarrow \infty\right)$ Regge-Teitelboim model.

\section{SUMMARY AND CONCLUSIONS}

The Israel Junction Conditions are known to be the major theoretical tool in dealing with gravitational layers, shells, domain walls, branes, etc. In this paper, based on a fundamental brane variation principle, as ingeniously prescribed by Dirac, we have actually challenged the tight structure of the traditional gravitation matching equations. Our main conclusion is that only a relaxed version of Israel Junction Conditions is dictated in fact by the underlying constrained gravity action. The Israel junction conditions have been modified in analogy to the Regge-Teitelboim modification of Einstein field equations. To be a bit more technical, the tensorial Eq.(27) has been traded for the scalar $\mathcal{K}$-contracted Eq.(34) and the conservation law Eq.(32). It is important to emphasize that we do not claim, or even suggest, that Israel matching equations are wrong; On the contrary, each and every solution of Israel matching equations is strictly respected. But following the present work, each such solution becomes a representative of a wider continuous family of solutions.

We have shown that the Dirac prescription for brane variation is as vital as ever when bulk/brane gravity is switched on, and if adopted, it may widely open the scope of modern brane theories. The prototype example is provided by the Randall-Sundrum theory which gets generalized accordingly. The more so, Regge-Teitelboim and Randall-Sundrum brane theories, which generalize General Relativity in two very different theoretical directions, appear in fact to be two faces of the one and the same unified brane theory. Exercising the option of including a brane curvature term in the Lagrangian, the unification scheme widen to also exhibit the Collins-Holdom and Dvali-Gabadadze-Porrati limits.

To appreciate the new formalism, the special case of a $Z_{2}$-symmetric unified brane cosmology has been studied in some details. Here, the central equation is the cubic Eq. (70) which allows us to conveniently navigate within the $\left(G_{4}^{-1}, G_{5}^{-1}, \omega\right)$ parameter space, with all special cases easily accessible. Not less important is Eq.(68), which allows a physicist, ignorant of the underlying unified brane theory, to recast the new equations into Einstein's field equations format, and thus interpret all deviations from General Relativity as a dark component. It remains to be seen if this has (or unfortunately does not have) anything to do with the real life dark matter problem. Other interesting sub-topics are currently under extensive investigation. In particular, some novel insight on the inflationary era, carrying the fingerprints of brane unification, will soon be released. And the same holds of course, naturally with high expectations, for the static radially symmetric case.

\section{Acknowledgments}

It is our pleasure to cordially thank Professors Eduardo Guendelman and David Owen for enlightening discussions and helpful comments. Special thanks to Shimon Rubin for sharing with us the 'ups and downs' moments of this research, and furthermore, for taking an active part in discussing several issues. 
[1] P.A.M. Dirac, Proc. Roy. Soc. A268, 57 (1962).

[2] W. Israel, Nuovo Cimento B44, 1 (1966). C. Lanczos, Phys. Zeils. 23, 539 (1922); Ann. der Phys. 74, 518 (1924). N. Sen, Ann. Phys. 73, 365 (1924). G. Darmois, Memorial des sciences mathematiques XX (1927).

[3] T. Regge and C. Teitelboim, in Proc. Marcel Grossman (Trieste), 77 (1975).

[4] L. Randall and R. Sundrum, Phys. Rev. Lett. 83, 3370 (1999). Phys. Rev. Lett. 83, 4690 (1999).

[5] G. Dvali, G. Gabadadze, M. Porrati Phys. Lett. B484, 112 (2000). G. Dvali, G. Gabadadze and M. Porrati, Phys. Lett. B485, 208 (2000). C. Deffayet, Phys. Lett. B502, 199 (2001). R. Cordero and A. Vilenkin, Phys.Rev. D65, 083519 (2002). T. Tanaka, Phys. Rev. D69, 024001 (2004). Y. Shtanov and A. Viznyuk, Class. Quant. Grav. 22, 987 (2005). A. Lue, Phys. Rep. 423, 1 (2006).

[6] H. Collins and B. Holdom, Phys. Rev. D62, 105009 (2000). H. Collins and B. Holdom, Phys. Rev. D62, 124008 (2000).

[7] R. Sundrum, Phys. Rev. D59, 085010 (1999). S.B. Giddings, E. Katz and L. Randall, J. High Energy Phys. 03, 023 (2000). J. Garriga and T. Tanaka, Phys. Rev. Lett. 84, 2778 (2000). C. Deffayet, Phys. Rev. D66, 103504 (2002). K.I. Maeda, S. Mizuno and T. Torii, Phys. Rev. D68, 024033 (2003) L. Gergely and R. Maartens, Phys. Rev. D71, 024032 (2005).

[8] P. Binetruy, C. Deffayet, and D. Langlois, Nucl. Phys. B565, 269 (2000). T. Shiromizu, K.I. Maeda and M. Sasaki, Phys. Rev. D62, 024012 (2000). R. Maartens, Phys. Rev. D62, 084023 (2000). P. Bowcock, C. Charmousis and R. Gregory, Class. Quant. Grav. 17, 4745 (2000). E.E. Flanagan, S.H.H. Tye and I. Wasserman, Phys. Rev. D62, 024011 (2000). E.J. Copeland, A.R. Liddle and J.E. Lidsey, Phys. Rev. D64, 023509 (2001). G. Kofinas, R. Maartens and E. Papantonopoulos, J. High Energy Phys. 10, 066 (2003).

[9] J.M. Maldacena, Theor. Math. Phys. 2, 231 (1998). R. Emparan, C. V. Johnson and R. C. Myers, Phys. Rev. D60, 104001 (1999). O. Aharony, S. S. Gubser, J. Maldacena, H. Ooguri and Y. Oz, Phys. Rep. 323, 183 (2000).

[10] F. Quevedo, Class. Quant. Grav. 19, 5721 (2002). E. Papantonopoulos, Lect. Notes Phys. 592, 458 (2002). D. Langlois, Prog. Theor. Phys. Supp. 148, 181 (2003). P. Brax and C. Van de Bruck, Class. Quant. Grav. 20, R201 (2003). R. Maartens, Living Rev. Rel. 7, 7 (2004). P. D. Mannheim, Brane-Localized Gravity (World Scientific Publishing Company, Singapore, 2005).

[11] K. Akama, Lect. Notes Phys. 176, 267 (1982). V.A. Rubakov and M.E. Shaposhnikov, phys. Lett. B125, 136 (1983). M. Visser, Phys. Lett. B159, 22 (1985). E.J.
Squires, Phys. Lett. B167, 286 (1986). S.K. Blau, E.I. Guendelman and A.H. Guth, Phys. Rev. D35, 1747 (1987).

[12] S. Deser, F.A.E. Pirani, and D.C. Robinson, Phys. Rev. D14, 3301 (1976).

[13] A. Davidson, Hadr. Jour. 21, 210 (1998). A. Davidson and D. Karasik, Mod. Phys. Lett. A13, 2187 (1998). A. Davidson, Class. Quan. Grav. 16, 653 (1999).

[14] M. Pavsic, Phys. Lett. A107, 66 (1985). G. Gibbons and D. Wiltshire, Nucl. Phys. B287, 717 (1987). V. Tapia, Class. Quan. Grav. 6, L49 (1989). D. Maia, Class. Quan. Grav. 6, 173 (1989). C. Romero, R. Tavakol and R. Zalatednikov, Gen. Rel. Grav. 28, 365 (1996). I.A. Bandos, Mod. Phys. Lett. A12, 799 (1997). M. Pavsic, Grav. Cosmol. 3, 305 (1997).

[15] D. Karasik and A. Davidson, Phys. Rev. D67, 064012 (2003). R. Capovilla, A. Escalante, J. Guven and E. Rojas (gr-qc/0603126).

[16] A. Davidson, D. Karasik, and Y. Lederer, Class. Quant. Grav. 16, 1349 (1999). A. Davidson, D. Karasik and Y. Lederer, hep-th/0112061 Phys. Rev. D72, 064011 (2005). R. Cordero and E. Rojas, Class. Quant. Grav. 21, 4231 (2004).

[17] B. Carter, Int .J. Theor. Phys. 40, 2099 (2001). A. Battye and B. Carter, Phys. Lett. B509, 331 (2001).

[18] D. Karasik and A. Davidson, Class. Quant. Grav. 211295 (2004).

[19] G. Hayward and J. Louko, Phys. Rev. D42, 4032 (1990). A. Davidson and D. Karasik, Phys. Rev. D60, 45002 (1999). S. Mukohyama, Class. Quant. Grav. 17, 4777 (2000); Phys. Rev. D65, 024028 (2002). J. Jezierski, J. Kijowski and E. Czuchry Phys. Rev. D65, 064036 (2002).

[20] H.A. Champlin and H.S. Reall, Nucl. Phys. B562, 133 (1999). C. Barcelo and M. Visser, Phys. Rev. D63, 024004 (2002). S.C. Davis, Phys. Rev. D67, 024030 (2003).

[21] G.W. Gibbons and S.W. Hawking, Phys. Rev. D15, 2752 (1977). R. Wald, in General Relativity (University of Chicago press, 1984).

[22] See for example, P.D. Mannheim, Prog. Part. Nucl. Phys. 56, 340 (2006).

[23] A. Davidson, Y. Lederer and D. Karasik, Proc. of The identification of dark matter (editors: N.J.C. Spooner and V. Kudryavtsev, World Scientific) p. 39 (2002); gr-qc/0111107

[24] A. Davidson, D. Karasik and Y. Lederer, Phys. Rev. D72, 064011 (2005).

[25] P. Binetruy, C. Deffayet, U. Ellwanger and D. Langlois, Phys. Lett. B477, 285 (2000). 